\title{
Integrin $\alpha 8$ Is Abundant in Human, Rat, and Mouse Trophoblasts
}

Reproductive Sciences

2017, Vol. 24(10) 1426-1437

(C) The Author(s) 2017

Reprints and permission:

sagepub.com/journalsPermissions.nav DOI: 10.1 |77//9337|9||6689597

journals.sagepub.com/home/rsx

(S)AGE

\author{
Sebastian Herdl', Hanna Huebner², Gudrun Volkert, PhD', \\ Ines Marek, MD', Carlos Menendez-Castro, MD', \\ Stephanie C. Noegel, MD', Matthias Ruebner, PhD²,

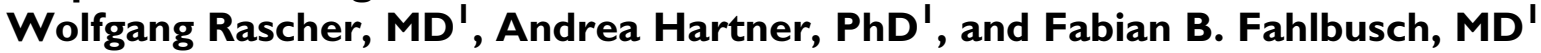

\begin{abstract}
Objective: Integrins exert regulatory functions in placentogenesis. Null mutation of certain integrin $\alpha$ subunits leads to placental defects with subsequent fetal growth restriction or embryonic lethality in mice. So far, the placental role of $\alpha 8$ integrin remains to be determined. Methods: Localization of $\alpha 8$ integrin and its ligands, fibronectin (FN) and osteopontin (OPN), was studied by immunohistochemistry in human, rat, and mouse placenta. The vascularization of the placental labyrinth layer of $\alpha 8$ integrindeficient mice was determined by CD3 I staining. In humans, $\alpha 8$ integrin expression was assessed via real-time polymerase chain reaction in healthy placentas, in the placental pathologies such as intrauterine growth restriction (IUGR), preeclampsia, and HELLP-syndrome (hemolysis, elevated liver enzymes, low platelet count), as well as in primary extravillous trophoblasts (EVT) and villous trophoblasts. Results: In humans, $\alpha 8$ integrin was detected in first and third trimester syncytiotrophoblast and EVT. Although OPN showed the same localization, FN was observed in EVT only. No expressional changes in $\alpha 8$ integrin were detected in the placental pathologies studied. Rodent placenta showed $\alpha 8$ integrin expression in giant cells and in the labyrinth layer. The localization of OPN and FN, however, showed species-specific differences. Knockout of $\alpha 8$ integrin in mice did not cause IUGR, despite some reduction in labyrinth layer vascularization. Conclusion: $\alpha 8$ Integrin is expressed in functional placental compartments among its ligands, OPN and/or FN, across species. Although this may point to a regulatory role in trophoblast function, our data from $\alpha 8$ integrin-deficient mice indicated only mild placental pathology. Thus, the lack of placental $\alpha 8$ integrin seems to be largely compensated for.
\end{abstract}

\section{Keywords}

integrins, Itga8, fibronectin, osteopontin, placenta, rodent, human, placental pathologies

\section{Introduction}

The maintenance of placental exchange and endocrine function is essential for fetal development and survival during pregnancy. Major structural deficiencies of this feto-maternal interface can result in early abortion. It can also lead to the development of placental pathologies, such as preeclampsia (PE), HELLP-syndrome (hemolysis, elevated liver enzymes, low platelet count), and intrauterine growth restriction (IUGR) ${ }^{1-3}$ all of which are associated with an increased rate of perinatal morbidity. ${ }^{4,5}$

During placentogenesis, the family of integrin matrix receptors plays an important role in adapting trophoblast cellular functions to the extracellular microenvironment via mediation of cell-matrix interactions. ${ }^{6,7}$

Integrins are transmembrane, heterodimeric glycoproteins consisting of $1 \alpha$ - and $1 \beta$-subunit. ${ }^{8}$ Their extracellular domain functions as a matrix adhesion molecule, while their intracellular domain mediates cellular functions like survival, migration, differentiation, and proliferation after ligand binding. ${ }^{9-12}$
The placental integrin expression pattern is dependent on the developmental stage and localization of the trophoblast. In the first trimester, trophoblasts are positive for $\alpha 6 \beta 4$ integrin, which is present at the basal surface of cytotrophoblasts (CTBs) throughout pregnancy and at the basal syncytial surface. From the second trimester, $\alpha 3 \beta 1, \alpha v \beta 3$, and $\alpha 5 \beta 1$ integrins are detected in CTB and in the syncytiotrophoblast (SCT), regulating mainly adhesion, cell turnover, and possibly syncytialization. ${ }^{13,14}$ Trophoblast differentiation into extravillous trophoblasts (EVTs) is accompanied by a shift in integrin

\footnotetext{
' Department of Pediatrics and Adolescent Medicine, University of ErlangenNürnberg, Erlangen, Germany

${ }^{2}$ Department of Gynaecology and Obstetrics, University of ErlangenNürnberg, Erlangen, Germany
}

\section{Corresponding Author:}

Fabian B. Fahlbusch, Department of Pediatrics and Adolescent Medicine, University of Erlangen-Nürnberg, Erlangen, Germany.

Email: fabian.fahlbusch@uk-erlangen.de 
expression: $\alpha 6 \beta 4$ integrin expression is lost, whereas $\alpha 1 \beta 1$, $\alpha 4 \beta 1, \alpha 5 \beta 1$, as well as $\alpha \mathrm{v} \beta 3$ and $\alpha 6 \beta 1$ integrins are upregulated to support EVT invasion and anchorage. ${ }^{14}$

The decisive role for integrins, in the development of the placenta, is emphasized by studies in mice with null mutations of some integrin $\alpha$ subunits. These animals showed a disruption of functional placental compartments, accompanied by an increased embryonic lethality. For example, Yang et $\mathrm{al}^{15}$ were able to show that a deficiency in the $\alpha 4$ integrin subunit resulted in a failure of the allantois to fuse with the chorion at $\mathrm{E} 8.5$.

In embryos deficient of $\alpha 7$ integrins, Welser et $\mathrm{al}^{16}$ found defects of placental vascular development. Placentas from E 9.5 and $\mathrm{E} 13.5$ showed a disruption of the infiltration of the spongiotrophoblast (ST) layer into the labyrinth, accompanied by fetal growth restriction.

In the placenta, most integrins have been thoroughly investigated. Up to now, the role of $\alpha 8$ integrin has not been studied. The $\alpha 8$ integrin subunit is known to dimerize exclusively with the $\beta 1$ integrin subunit. ${ }^{17}$ Ligands of $\alpha 8 \beta 1$ integrin are fibronectin (FN) and osteopontin (OPN), among others. ${ }^{18-20}$ Both FN and OPN are abundantly expressed in the placenta: FN expression is detected in the villous stroma and the decidua. ${ }^{21}$ Osteopontin is a major constituent of the uteroplacental compartment and known as a marker of decidualization. ${ }^{22}$

$\alpha 8$ Integrin-deficient mice frequently show abnormalities in embryonic development, leading to uni- or bilateral renal agenesis in part of the animals and to a malformation of stereocilia in the inner ear. ${ }^{23,24}$ Moreover, $\alpha 8$ integrin-deficient mice are highly susceptible to mechanical or inflammatory renal or vascular organ damage. ${ }^{25-27}$ Thus, $\alpha 8$ integrin seems to play a pivotal role in maintaining structural and functional tissue integrity in these organs.

The cellular effects of $\alpha 8 \beta 1$ integrin binding are strongly cell type dependent. It inhibits migration and stimulates proliferation in smooth muscle cells and in human intestinal crypt cells. In the latter, overexpression of $\alpha 8 \beta 1$ integrin also induces cell differentiation. ${ }^{28-31}$ In contrast, knockdown of $\alpha 8 \beta 1$ integrin increases proliferation in mesangial cells. ${ }^{32}$ Similarly, opposing observations were made upon investigating cell survival. Acute knockdown of $\alpha 8 \beta 1$ integrin lead to resistance against anoikis in intestinal cells, but its expression guarded neuroendocrine cells from apoptosis. ${ }^{33,34}$

In light of the findings that $\alpha 8$ integrin regulates cell survival, proliferation, differentiation, and migration in many cell types and that the placenta is an organ in which apoptosis, proliferation, differentiation, and migration of trophoblast cells occur tightly regulated throughout pregnancy, we hypothesized that $\alpha 8$ integrin is expressed in placental compartments, which could argue for a role of $\alpha 8$ integrin for placental development and homeostasis. Therefore, we analyzed the expression and localization of $\alpha 8$ integrin and its ligands, FN and OPN, in human and rodent placenta. Moreover, we studied $\alpha 8$ integrin expression in placental pathologies (PE, HELLP, IUGR) and investigated the placentas of $\alpha 8$ integrin-deficient mice.

\section{Materials and Methods}

\section{Animal Procedures}

All animal procedures were carried out in accordance with the Guide for the Care and Use of Laboratory Animals published by the US National Institutes of Health (NIH Publication No. 85-23, revised 1996) and were approved by the local government authorities (Regierung von Mittelfranken, approval numbers AZ \# 542531.31-12/06 and AZ \# 54-2532.1-17/08) after evaluation of the local government's review board for animal research ethics. All surgeries were performed under isoflurane anesthesia and all efforts were made to minimize suffering. The animals were housed in a room maintained at $22^{\circ} \mathrm{C} \pm 2^{\circ} \mathrm{C}$, exposed to a 12-hour dark/ light cycle, and were allowed unlimited access to standard chow and tap water. The mouse model of $\alpha 8$ integrin-deficient (Itga $8^{-/}$) mice was previously described. ${ }^{25} \mathrm{We}$ used Itga $8^{-/-}$mice and their wild-type (WT) litters from a heterozygous breeding for mating. This mouse strain is kept on a mixed genetic background $129 \mathrm{~Sv} /$ C57BL6. For collection of Itga $8^{-1}$ placentas, female Itga $8^{-1-}$ and male Itga $8^{+/-}$were mated; for collection of WT placentas, female WTs and male Itga $8^{+/-}$were mated. The placentas were collected at day 19.5 of gestation and were genotyped. Seven WT placentas and 5 Itga $8^{-/}$placentas from different litters were used for expression analysis. The neonatal weights were assessed from 22 WT (from 5 different litters) and 16 Itga $8^{-/-}$(from 5 different litters). Mouse placentas were obtained from these mice at day 17.5 of pregnancy. Pregnant female Wistar rats were obtained from Charles River (Sulzfeld, Germany). Rat placentas were obtained following maternal sacrifice via bleeding in deep anesthesia at day 19 of pregnancy. All rodent placentas were fixed in methyl Carnoy or ice-cold $4 \%$ paraformaldehyde for embedding in paraffin or were snap frozen for messenger RNA (mRNA) preparation.

\section{Human Placental Tissue}

This study was conducted in accordance with principles outlined in the Declaration of Helsinki ${ }^{35}$ and was approved by the institutional ethics committee of the medical faculty of the University of Erlangen-Nürnberg (\#2625-02/28/02 and \#2180-05/05/ $04)$. Written informed consent was given by every patient.

Placental sampling and tissue handling were described earlier in detail. $^{36,37}$ For immunohistochemical analysis, fresh samples of human placenta were excised immediately after placental delivery, fixed in $4 \%$ formaldehyde, and embedded in paraffin. We analyzed tissue sections of healthy human appropriate for gestational age (AGA) placentas $(n=5)$ and of pregnancies complicated by IUGR, PE, and HELLP ranging from 36 gestational weeks to term $(n=5$ each). Cases of IUGR were not complicated by PE and vice versa. The diagnosis of IUGR, PE, and HELLP was based on generally accepted criteria outlined previously. ${ }^{37,38}$ Moreover, placental sections of first-trimester abortions $(n=4)$ were examined (kind gift of the Department of Pathology at the University of Erlangen).

For quantitative polymerase chain reaction (PCR) gene expression analysis, a separate set of placental samples containing third trimester AGA $(\mathrm{n}=25)$, IUGR $(\mathrm{n}=18)$, PE $(\mathrm{n}=$ $18)$, and HELLP $(n=8)$ samples was used. These samples were 
Table I. Murine Primer Pairs.

\begin{tabular}{|c|c|c|}
\hline & Forward & Reverse \\
\hline $\operatorname{ltga} 5$ & 5'-TCG GAG CAA CAG TTC GGG-3' & 5'-GTG GAG CAC ATG CCA AGA TG-3' \\
\hline $\operatorname{ltga2}$ & $5^{\prime}$-CTG TGA TCC AGC AAT GCA ATG-3' & $5^{\prime}$-ACT GCT ATG CCG AAC CTC AGT- $3^{\prime}$ \\
\hline Itga6 & $5^{\prime}$-AAC ATC AGG TGC CCA CTG C- $3^{\prime}$ & 5'-AAG AAC GAG AGA GGC CTT GCT-3' \\
\hline CD31 & $5^{\prime}$-TGC GGT GGT TGT CAT TGG-3' & $5^{\prime}$-TGT TTG GCC TTG GCT TTC CG-3' \\
\hline
\end{tabular}

obtained in a standardized sterile procedure immediately after placental delivery. A $2 \times 2 \mathrm{~cm}$ segment, total depth ranging from basal plate to chorionic membrane, was excised at the central region of the placenta using a scalpel blade. Macroscopic calcifications were avoided. Amniotic membranes and basal plate were removed. Samples were immediately snap frozen in liquid nitrogen and stored at $-80^{\circ} \mathrm{C}$ until further processing.

In a third set of experiments, primary trophoblast cells were isolated from uncomplicated term pregnancies after elective cesarean section as previously described in detail. ${ }^{36,39}$ We isolated decidual stroma cells (DSCs), EVTs, and villous cytotrophoblasts (VT) from 3 total AGA placentas. The procedures were carried out under sterile conditions. Protocols for immunomagnetic bead separation of VT and EVT isolation have been described in detail elsewhere. $^{36,40}$ In short, for the fractionation of EVT, placental basal plate was grossly removed, minced, and subjected to enzymatic digestion involving trypsin, DNase I (Sigma-Aldrich, Taufkirchen, Germany), and dispase (BD Biosciences, Heidelberg, Germany). After filtration and centrifugation, the resuspended cells were layered over lymphocyte separation medium 1077(Sigma-Aldrich). After erythrocyte lysis, cells were resuspended in MACS buffer (Miltenyi Biotec, Bergisch Gladbach, Germany) containing DNase I and subjected to Fc-blocking (Miltenyi Biotec), followed by incubation with anti-human leukocyte antigen (HLA)-G-phycoerythrin (PE) antibody (clone 87G; Biolegend, Fell, Germany). Subsequently, cells were incubated with anti-PE magnetic beads (Miltenyi Biotec), washed and loaded on magnetized LS columns (Miltenyi Biotec), and the flow-through discarded. Isolated EVTs were HLA-G positive and CD45/CD3/CD56 negative, as shown by multiple FACS (FACSCalibur; BD Biosciences) analyses. ${ }^{40}$

Fractionation and cultivation of human VT was performed based on the trypsin-DNase-dispase/Percoll method ${ }^{41,42}$ in conjunction with negative immunomagnetic bead separation (Miltenyi Biotec) via the PE-labeled surface marker antibodies HLAABC (clone W6/32; Biolegend, London, United Kingdom) and CD45 (clone BRA-55; Sigma-Aldrich), as previously described in detail. ${ }^{40}$ Percoll came from Sigma-Aldrich. Isolated VTs were $99 \%$ HLA-ABC and CD45 negative. ${ }^{40}$ Isolation of DSCs from placenta via selective adhesion was carried out by modifying the protocols of Frank et $\mathrm{al}^{43}$ and Lockwood et $\mathrm{al}^{44}$, as previously published in detail. ${ }^{39}$ In short, enzymatic digestion of minced placental basal plate was carried out with collagenase type I (Gibco-BRL, Eggstein, Germany) and DNase I (Sigma-Aldrich). Following erythrocyte lysis, the filtrated and washed cells were seeded. Decidual stromal cells were separated from contaminating cells via their reduced adherence and purified by multiple passaging. Purity was assessed after the third passage via FACS analysis (FACSCalibur) using the following antibodies: vimentin (clone V9; Dako, Hamburg, Germany), CD45 (Caltag Laboratories, Hamburg, Germany), CD9 (Pharmingen, San Diego, California), CD31, and CD14 (Immunotec, Quebec, Canada).

\section{Gene Expression Analysis}

Gene expression analysis was performed as previously described. ${ }^{37}$ In short, for RNA purification, peqGold TriFast reagent (Peqlab, Erlangen, Germany) was used. Following RNA pretreatment with DNase I (Sigma-Aldrich), complementary DNA (cDNA) synthesis was conducted using the high-capacity cDNA reverse transcription kit (Life Technologies, Darmstadt, Germany) in a thermal cycler (ABI2720). Quantification of $\alpha 8$ integrin mRNA expression was achieved by quantitative real-time PCR analysis using the SYBR Select Master Mix (Life Technologies) with 18srRNA as housekeeping gene. Measurements were performed in duplicates. Primers were designed using Primer 3 software. ${ }^{45}$ The following primers were used for human tissue (Eurofins Genomics, Ebersberg, Germany): ITGA8 forward 5'-GCGCAGTCCT GAAAGTCAGG-3', reverse 5'-TCTGGAGGAAGGTGT GGGC- $3^{\prime}$ and 18srRNA forward $5^{\prime}$-GCAATTATTCCCCAT GAACG- $3^{\prime}$, reverse $5^{\prime}$-GGCCTCACTAAACCATCCAA-3' . For mouse tissue, primers are listed in Table 1.

\section{Immunohistochemistry}

Unless stated otherwise, all materials came from SigmaAldrich. Tissue fixation in methyl Carnoy and paraformaldehyde and embedding in paraffin were performed as previously described. ${ }^{46,47}$ After cutting 4- $\mu \mathrm{m}$ paraffin sections with Leica CM3050S microtome (Leica Instruments, Wetzlar, Germany), sections were deparaffinized in xylol and rehydrated by bathing in decreasing concentrations of alcohol. The endogenous peroxidase activity was blocked for 20 minutes at room temperature with $3 \% \mathrm{H}_{2} \mathrm{O}_{2}$ in methanol.

For Itga 8 staining, human and rat placental sections were incubated with $1 \%$ bovine serum albumin (BSA) in Tris-buffered saline (TBS; $1 \% \mathrm{BSA} / \mathrm{TBS}$ ) at $37^{\circ} \mathrm{C}$ for 30 minutes, whereas sections of mice placenta were incubated using fetal calf serum (FCS) under the same conditions.

For the FN staining of all sections and for the OPN staining of only rat and human sections, they were coated with $1 \% \mathrm{BSA} /$ 
TBS. Murine placenta first needed an avidin-biotin block (SP2001) followed by $1 \% \mathrm{BSA} / \mathrm{TBS}$ at room temperature for 30 minutes. Afterward, the sections were coated with the primary antibody and incubated overnight at $4{ }^{\circ} \mathrm{C}$. The polyclonal goat antibody to Itga8 (AF4076; R\&D systems Inc, Minneapolis, Minnesota) was used at a dilution of 1:50 (human, rat) or 1:100 (murine). For the FN staining, a dilution of 1:100 (murine) or 1:500 (human, rat) of polyclonal rabbit antibody (ab2413; Abcam, Cambridge, United Kingdom) was used. The polyclonal rabbit antibody to OPN (ab33046; Abcam) was used at a dilution of 1:50. Sections were layered with the secondary antibody (biotin-conjugated, horse antigoat immunoglobulin $\mathrm{G}$ (IgG) [BA-9500] or goat antirabbit IgG [BA-1000] at room temperature for 30 minutes at a dilution of 1:500, except for FN staining of human and rat placenta, where a dilution of 1:250 was used. Subsequently, sections were incubated with horseradish peroxidase avidin D (dilution 1:2000; A-2004) at room temperature for 30 minutes and ImmPACT DAB peroxidase substrate kit (for human and rat sections; SK-4105) or peroxidase substrate 3,3'-diaminobenzidine (DAB) kit (for murine sections; SK-4100; all Vector Laboratories Inc, Burlingame, California), respectively. ${ }^{26,48}$ Hematoxylin (Merck, Darmstadt, Germany) was used as a counterstaining dye. Between each step, sections were washed with TBS. For examination of general placental histology, murine placenta was stained with PAS reagent.

For staining of trophoblasts in human first trimester serial sections, we used a mouse monoclonal pan-cytokeratin antibody (clone 80, 1:500; Kamiya Biomedical Co, Seattle) at a dilution of 1:200 in conjunction with the labeled streptavidin binding (LSAB) + horseradish peroxidase (HRP) kit (Dako) and hematoxylin as per the manufacturer's instructions, as previously published. ${ }^{49}$

Sections were analyzed using a Leitz Aristoplan microscope (Leica Microsystems, Wetzlar, Germany). The description of rodent placental anatomy was based on nomenclature published by others before. ${ }^{50-52}$

\section{Determination of Labyrinth Vascularity}

To stain vascular structures within the labyrinth, CD31 (Pecam1) immunofluorescent (IF) staining was performed. Paraffin sections were deparaffinized and rehydrated, and endogenous peroxidase activity was blocked as described above. Unmasking was performed with cooking in target retrieval solution (TRS; Dako) for 20 minutes and cooling in TRS for another 10 minutes. After incubating with $\mathrm{FCS}$ at $37^{\circ} \mathrm{C}$ for 30 minutes, the sections were coated with rabbit polyclonal anti-CD31 antibody (dilution 1:50, ab 28364; Abcam) and incubated overnight at $4^{\circ} \mathrm{C}$. After washing, slides were incubated with the secondary fluorescent antibodies (Alexa Fluor 555 red at a dilution of 1:500 for 2 hours and 4',6-diamidino-2-phenylindole (DAPI, blue) at a dilution of 1:2000 for 2 minutes, all from Life Technologies, Darmstadt, Germany) and embedded in Mowiol. Immunofluorescent sections were analyzed using a Nikon Eclipse 80i microscope (Nikon GmbH, Düsseldorf, Germany).

To screen for potential defects of vascular development in the labyrinth layer ${ }^{16}$ of $\operatorname{Itga} 8^{-/-}$mice, the percentage of vascular elements (CD31 positive) within the murine labyrinth was analyzed over 6 random fields of 2 IF slides per animal ( 5 Itga $8^{-/-}$ vs $5 \mathrm{WT})$ at $\times 400$ magnification using ImageJ software $(1.48 \mathrm{v}$; $\mathrm{NIH}^{53}$ ). For standardization of our stereologic analysis, we chose those representative placental cross sections (30 $\mu \mathrm{m}$ apart) with the largest vertical diameter in each animal.

\section{Statistical Analysis}

Results were expressed as mean \pm standard error of the mean (SEM) unless stated otherwise. Statistical analyses were performed using GraphPad Prism Version 4.0c (GraphPad Software, San Diego, California). Groups were compared using 2-sided unpaired $t$ test. A $P$ value of $<.05$ was considered statistically significant.

\section{Results}

\section{Localization of $\alpha 8$ Integrin in Human First Trimester Placenta}

ITGA8 staining was evident in extravillous and villous placental compartments (Figure 1a). Extravillous cells (ie, DSCs and EVT) both showed ITGA8 antibody staining (Figure 1b). Extravillous trophoblasts stained both ITGA8 and pan-cytokeratin positive in serial sections of first trimester placenta (Figure S4 a, c). In the first trimester villi, $\alpha 8$ integrin was majorly localized in the SCT showing a basoapical staining pattern, with minor ITGA8 staining in the adjacent villous trophoblast (VT, Figure 1c). Similar to EVT, SCT of the first trimester stained ITGA8 and pan-cytokeratin positive (Figure S4). We found strong FN immunoreactivity in the basal plate matrix surrounding EVT (Figure 1e). Villous stroma (Figure 1d), decidual stroma (Figure 1e), VT, and SCT (Figure 1f) stained largely FN negative. Both extravillous (DSC and EVT; Figure 1g and $\mathrm{h}$ ) and villous compartments (Figure 1g and i) stained positive for OPN. The SCT showed a basoapical OPN staining pattern (Figure 1i).

\section{Localization of $\alpha 8$ Integrin in Healthy Human Third Trimester Placenta and Placental Pathologies}

Similar to our findings in human first trimester placenta, immunostaining of $\alpha 8$ integrin was evident in all placental compartments of the third trimester placenta (Figure 2a-c). Basal plate EVT showed a strong ITGA8 immunoreactivity with a predominant membranous staining pattern (Figure $2 b$ ), even more pronounced than in the first trimester EVT (Figure 1b). In the third trimester placental villi, the SCT stained positive for ITGA8, whereas VT stained only weakly for ITGA8 (Figure 1c). Similar to the findings in first trimester placenta (Figure 1d), basal plate EVT themselves were negative, but there was a strong extracellular immunoreactivity to $\mathrm{FN}$ in the basal plate (Figure $2 \mathrm{~d}$ and e). As seen in the first trimester placenta (Figure 1f), SCT and VT stained FN negative (Figure 2f). Osteopontin staining pattern at term reflected findings from the first trimester (Figure 1g-i), with positive EVT (Figure 2g-h), SCT (Figure $2 \mathrm{~g}$ and i) and VT (Figure 2i). As described by others, ${ }^{54}$ 


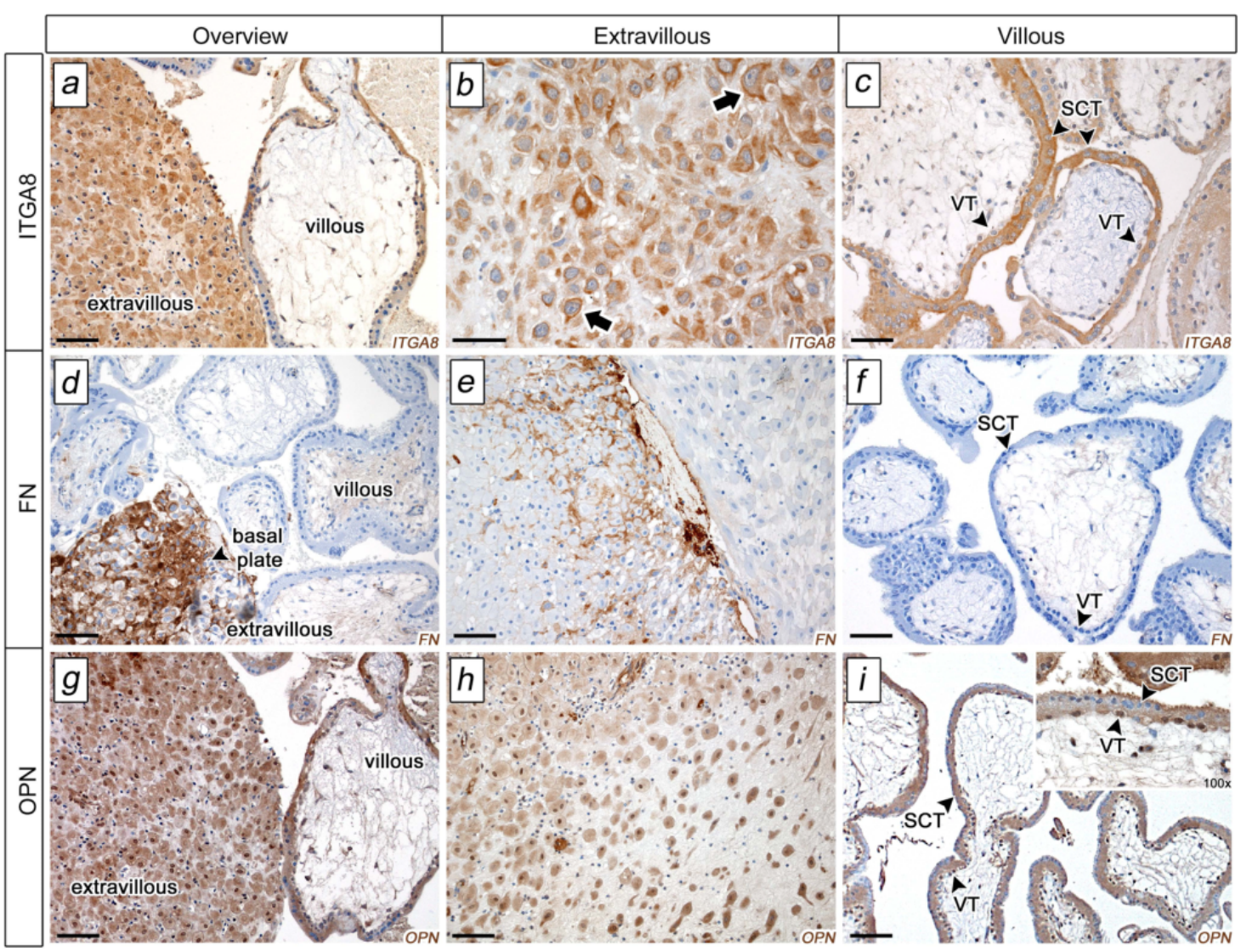

Figure I. Localization of $\alpha 8$ integrin in human first trimester placenta. Localization of $\alpha 8$ integrin (ITGA8, a-c) and its ligands fibronectin (FN, $\mathrm{d}-\mathrm{f}$ ) and osteopontin (OPN, g-i; immunohistochemical peroxidase stain with DAB detection, representative for $n=4$ ). a, $d$, and $g$ give an overview of the respective staining showing all placental compartments. b, e, and h show the extravillous compartment at a higher magnification. c, $f$, and $i$ show syncytiotrophoblast (SCT) and villous trophoblasts (VT) at a higher magnification. The bar equals $100 \mu m$. Positive stainingbrown; nuclei counterstained with hematoxylin—blue; black arrows indicate membranous staining pattern (b).

we also found positive OPN membrane staining of fetal erythrocytes, while maternal blood cells showed no OPN immunoreactivity (data not shown).

\section{Expression of $\alpha 8$ Integrin in Primary Human Trophoblasts and Placental Pathologies}

Analysis of ITGA8 mRNA expression in primary human third trimester EVT and VT by real-time PCR showed a significantly higher ITGA8 expression in EVT compared to VT (Figure 2j), which is in accordance with our findings from the histological analyses. The comparison of healthy and pathological placental tissue showed no significant difference in ITGA8 expression (Figure $2 \mathrm{k}$ ). This finding was in line with our immunohistochemical analyses of placental ITGA8 expression in these entities, where we did not detect clear-cut differences in ITGA8 staining of pathological and control placentas (data not shown).

\section{Localization of $\alpha 8$ Integrin in Rat Term Placenta}

Maternal decidua and trophoblast giant cells (Figure 3b) stained Itga 8 positive, whereas the ST (Figure 3a) and the labyrinth zone (LZ, Figure 3c) showed no Itga8 immunoreactivity.

All placental compartments of the rat placenta stained FN positive (Figure 3d and e), except for the LZ (Figure 3f). Fibronectin staining appeared in the extracellular matrix surrounding GC and cells of the ST layer (Figure 3e), similar to staining patterns in the basal plate surrounding human EVT (Figure $2 \mathrm{~d}$ and e). Osteopontin expression was predominantly found in GC and the adjacent ST (except for OPN-negative glycogen cells; Figure $3 \mathrm{~h}$ ), with a cytoplasmatic staining pattern (Figure 3h). In contrast to the negative FN stain (Figure 3f), the rat LZ showed strong OPN immunoreactivity (Figure 3i), with a pronounced OPN staining in mononuclear sinusoidal trophoblast giant cells (sTGCs; Figure 3i, magnification).

\section{Localization of $\alpha 8$ Integrin in Mouse Term Placenta}

Similar to the findings in rat placenta, we observed Itga8 immunoreactivity in the decidua and in GC (Figure 4a and b). However, Itga 8 staining pattern in the mouse was slightly different from the rat (Figure 3c). In contrast to the rat, the maternal decidua of mice was Itga 8 negative (Figure 4a), the ST 


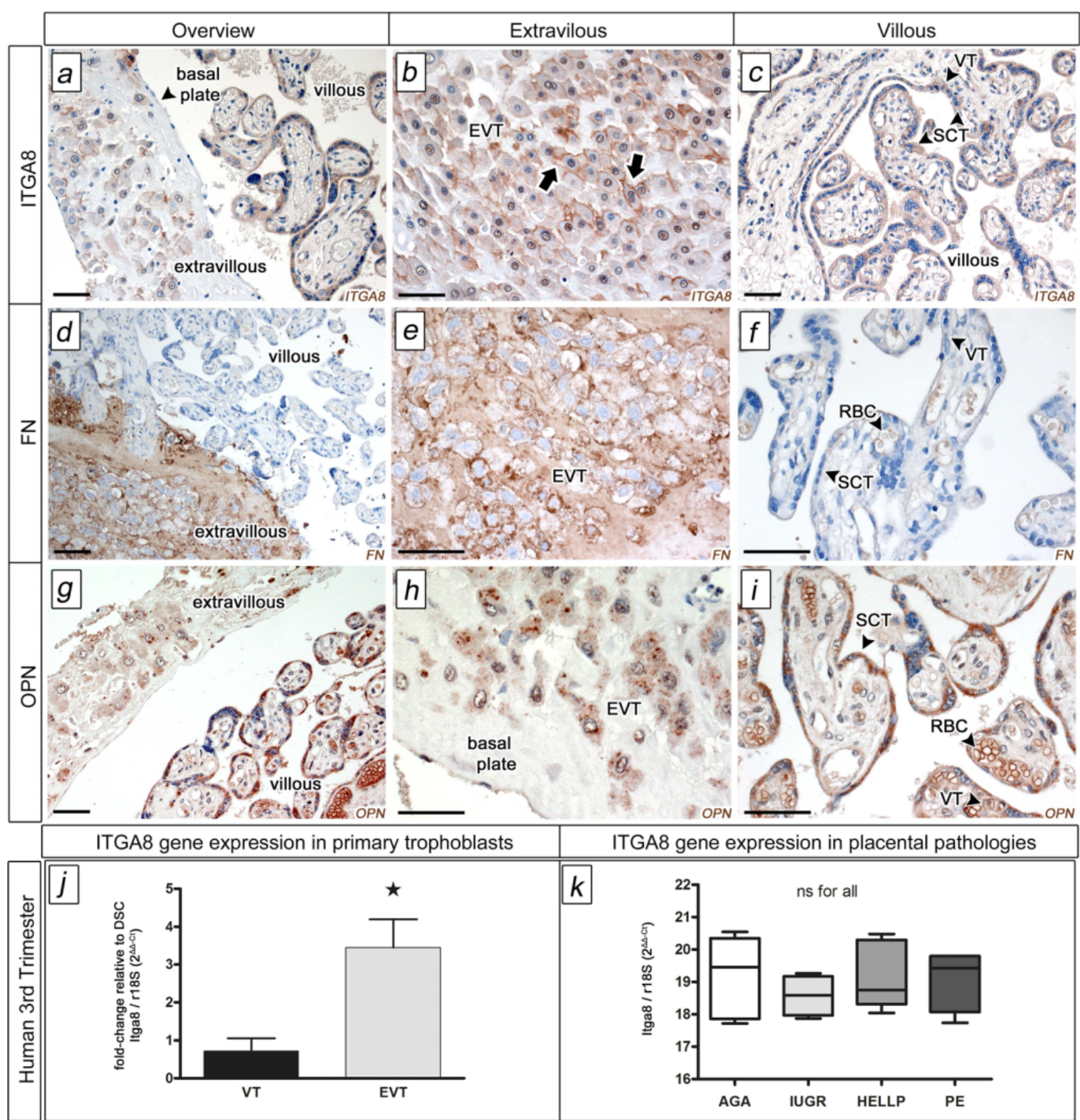

Figure 2. Localization and expression of $\alpha 8$ integrin in healthy human third trimester placenta and placental pathologies. Localization of $\alpha 8$ integrin (ITGA8, a-c) and its ligands FN (d-f) and OPN (g-i) in human third trimester placenta (immunohistochemical peroxidase stain with DAB detection, representative for $\mathrm{n}=5$ ). a, $d$, g give an overview of the respective staining showing all placental compartments. b, e, and $h$ show EVT at a higher magnification. $c, f$, and i show SCT and VT at a higher magnification. $j$ and $k$ show the expression of $\alpha 8$ integrin in primary human trophoblasts (isolated from $n=3$ placentas, each) and placental pathologies: AGA $(n=25)$, IUGR $(n=I 8)$, PE $(n=18)$, and HELLP $(n=8)$. $* P<.05$, The bar equals $100 \mu \mathrm{m}$; black arrows indicate membranous staining pattern (B). Positive staining-brown; nuclei counterstained with hematoxylin—blue. AGA indicates appropriate-for-gestational age; DSC, decidual stroma cells; EVT, extravillous trophoblasts; FN, fibronectin; IUGR, intrauterine growth restriction; PE, preeclampsia; OPN, osteopontin; RBC, red blood cell; SCT, syncytiotrophoblast; VT, villous trophoblasts.

showed a very weak Itga8 immunoreactivity (Figure $4 b$ ), and the LZ (Figure 4c) stained Itga8 positive. Examining the LZ at a higher magnification (Figure 4c), we found strong Itga 8 immunoreactivity in its syncytial layers (Syn), with negative Itga8 staining of fetal vessel endothelium and mononuclear sTGCs (Figure 3c). As seen in the rat (Figure 3d-f), extracellular FN deposition in the connective tissue surrounding trophoblast GC (Figure 4d and e) and ST (Figure 4e) was found. In the mouse, positive FN immunoreactivity of the ST was more sporadic when compared to the rat. Glycogen-containing cells were FN negative (Figure 4e). In contrast to the rat, we detected a strong FN staining in the mouse LZ (Figure 4f, magnification) with a specific FN immunoreactivity in its Syn. Similar to our findings in the rat placenta (Figure $3 \mathrm{~g}-\mathrm{i}$ ), the murine placenta (Figure 4g-i) expressed OPN in GCs and the adjacent ST. Osteopontin immunoreactivity of the latter seemed more pronounced in mice (Figure $4 \mathrm{~h}$ ). Glycogen cells remained OPN negative (Figure 4h), whereas the LZ showed 


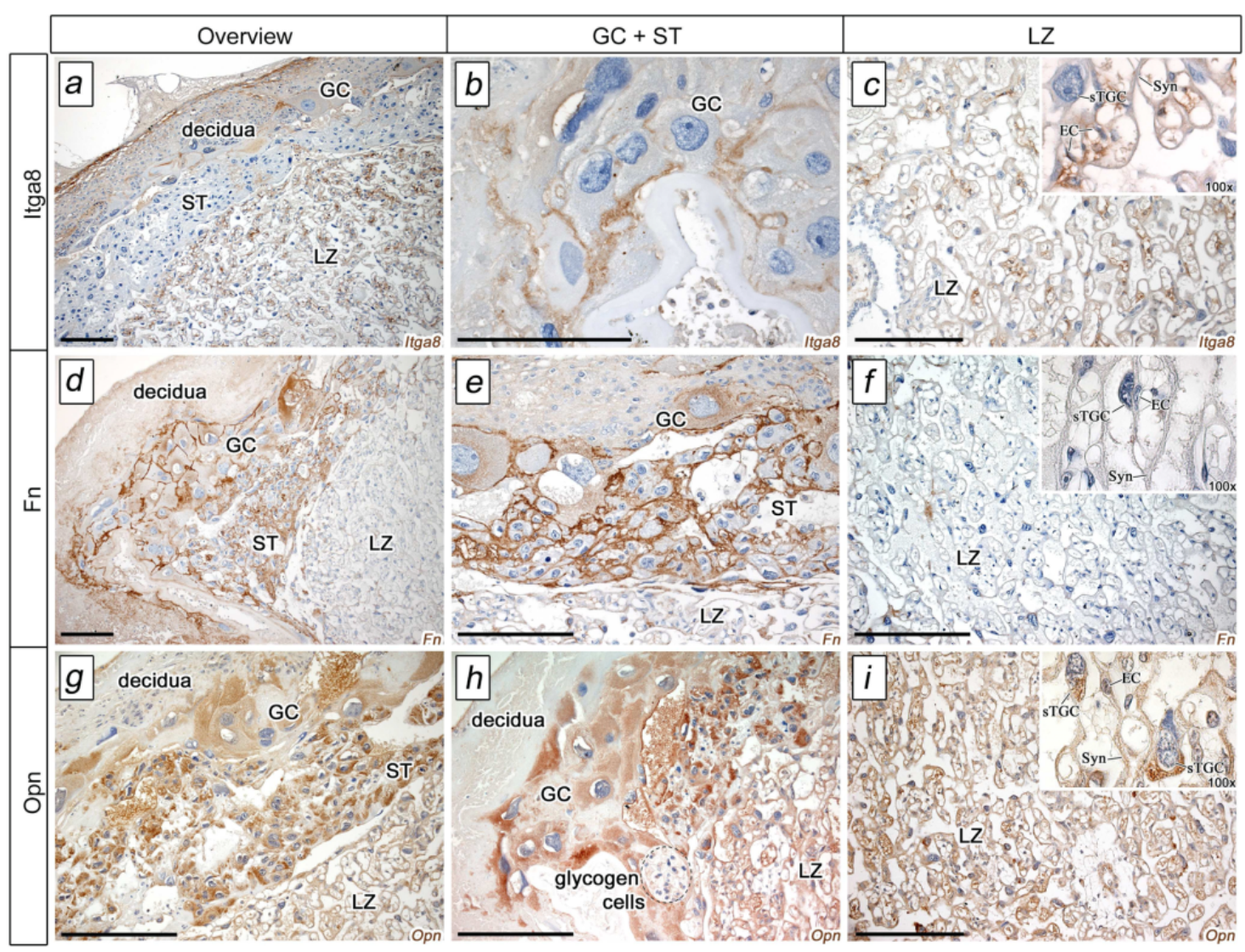

Figure 3. Localization of $\alpha 8$ integrin in rat term placenta. Localization of $\alpha 8$ integrin (Itga8, a-c) and its ligands FN (d-f) and OPN (g-i) in rat term placenta (immunohistochemical peroxidase stain with DAB detection, representative for $n=6$ ). a, $d$, and g give an overview of the respective staining showing all placental compartments. b, e, and h show GCs and spongiotrophoblast (ST) at a higher magnification. c, f, and i show staining in the LZ at a higher magnification. The bar equals $100 \mu \mathrm{m}$. Positive staining-brown; nuclei counterstained with hematoxylin-blue. EC indicates endothelial cell; FN, fibronectin; GCs, giant cells; LZ, labyrinth zone; OPN, osteopontin; sTGC, mononuclear sinusoidal trophoblast giant cell; Syn, syncytial trophoblast layers.

some OPN immunoreactivity (Figure $4 \mathrm{i}$ ). As seen in the rat (Figure 3i), sTGCs showed a pronounced positive OPN staining (Figure 4i).

\section{Determination of Labyrinth Vascularity in $\alpha 8$ Integrin-Deficient Mice}

Comparison of murine Itga8 knockout $\left(\mathrm{Itga}^{-1-}\right.$ ) and WT placentas did not reveal any alterations in size or the relation of the functional compartments to each other (data not shown). However, when examining the labyrinth layer in more detail, some reduction in vascularity was evident, which was assessed by quantifying labyrinthine CD31 immunoreactivity (Figure 5a). On the other hand, CD31 mRNA expression was not different in Itga $8^{-/-}$and WT placentas (Table 2). To test if placentas lacking Itga8 are insufficient, we compared birth weights of Itga $8^{-1-}$ and WT mice. No differences in birth weights were detected (Figure $5 \mathrm{~b}$ ). We hypothesized that in the placenta, the lack of Itga 8 might be compensated for by other integrin chains. Expression analysis of candidate integrin chains Itga5, ItgaV,
Itga2, and Itga6 revealed no differences in expression levels in Itga $8^{-1-}$ and WT placentas (Table 2).

\section{Discussion}

We determined the expression of $\alpha 8$ integrin and its tripeptide Arg-Gly-Asp (RGD) sequence containing ligands FN and $\mathrm{OPN}^{18,20}$ in human and rodent placenta. Prominent immunostaining for $\alpha 8$ integrin was detected in human EVT and SCT with weaker staining of villous CTB. Staining for $\alpha 8$ integrin in human placenta and for FN and/or OPN revealed common patterns in the examined placental compartments, suggesting possible interactions of $\alpha 8$ integrin with these ligands.

Tightly regulated expression of integrins is a prerequisite for proper placental development and governs the turnover of trophoblast cells and the invasion of EVT into the decidua, where integrins regulate spiral artery tone or establish anchorage of placental villi. ${ }^{55,56}$ It is known that this invasion process is accompanied by the loss of $\alpha 6 \beta 4$ integrin and induction of $\alpha 5 \beta 1$ and $\alpha 1 \beta 1$ integrins. ${ }^{55,56} \mathrm{~A}$ dysregulation of this integrin shift is associated with pathologic pregnancy outcome, such as 


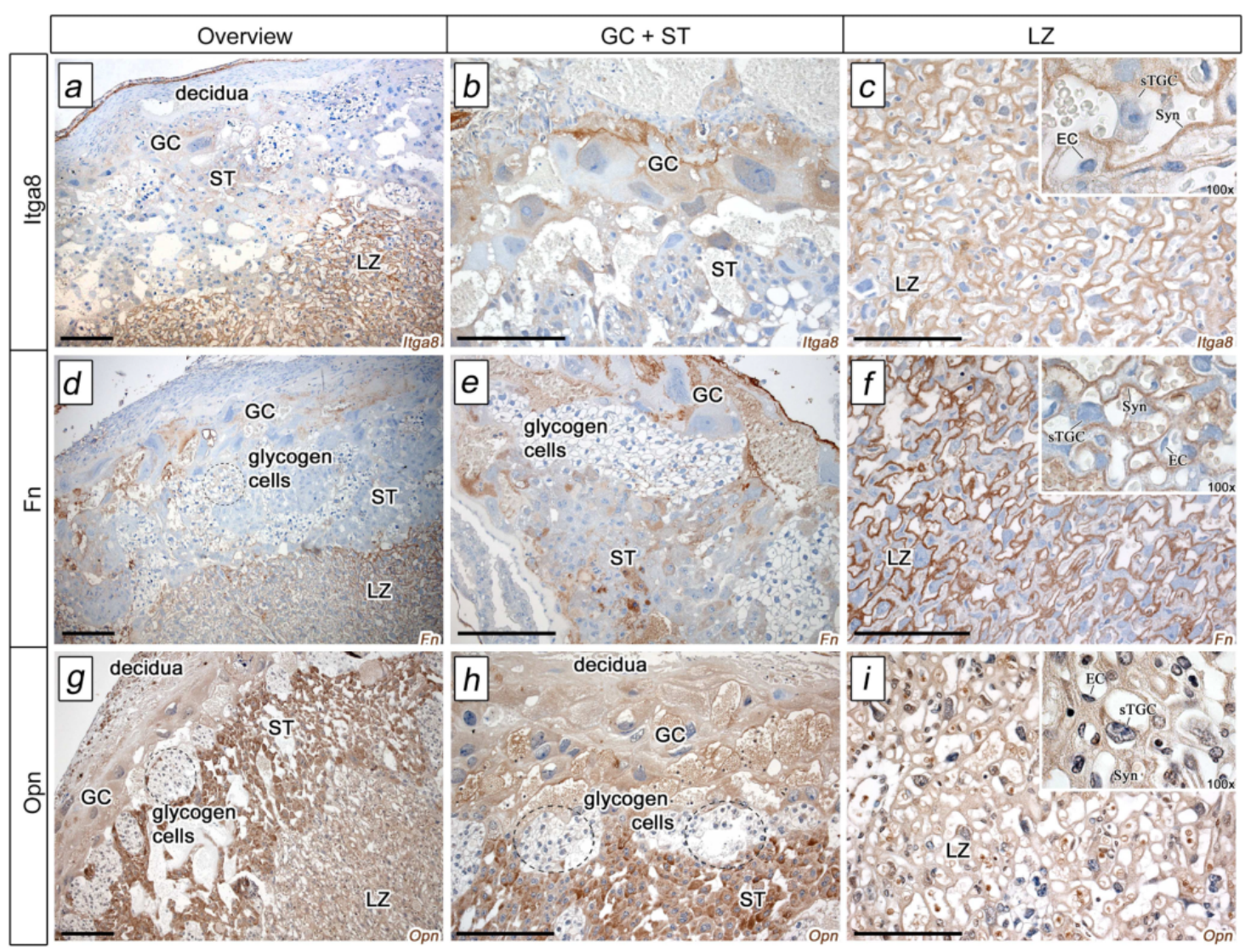

Figure 4. Localization of $\alpha 8$ integrin in mouse term placenta. Localization of $\alpha 8$ integrin (Itga8, a-c) and its ligands FN (d-f) and OPN (g-i) in mouse term placenta (immunohistochemical peroxidase stain with DAB detection, representative for $n=5$ ). a, $d$, and $g$ give an overview of the respective staining showing all placental compartments. b, e, and h show GC and ST at a higher magnification. c, f, and i show staining in the LZ at a higher magnification. The bar equals $100 \mu \mathrm{m}$. Positive staining-brown; nuclei counterstained with hematoxylin-blue. EC indicates endothelial cell; FN, fibronectin; GCs, giant cells; LZ, labyrinth zone; OPN, osteopontin; sTGC, mononuclear sinusoidal trophoblast giant cell; Syn, syncytial trophoblast layers; ST, spongiotrophoblast.

PE, characterized by a shallow CTB invasion of the placental bed and of uterine vessels. ${ }^{57-59}$ It is therefore conceivable that $\alpha 8$ integrin might also play a role in the regulation of trophoblast turnover and/or EVT invasion and anchorage in the decidua. Studies in several cell types proved a contribution of $\alpha 8$ integrin to the regulation of apoptosis, ${ }^{26,34}$ proliferation, ${ }^{29,30,32}$ adhesion, ${ }^{32}$ and migration. ${ }^{32,60}$

In the human placenta, $\alpha 8$ integrin expression was mainly located in EVT and SCT, whereas FN was localized in close vicinity of EVT in the basal plate. This is in accordance with previous studies where FN was specifically secreted by migrating basal plate EVT. ${ }^{61,62}$ This argues for a possible interaction of FN and $\alpha 8$ integrin in human EVT. In line with this finding, the amount of released FN is strongly influenced by integrin expression of trophoblast cells. ${ }^{63}$ Osteopontin was detected in DSCs, EVT, SCT, and VT arguing for possible interactions with $\alpha 8$ integrin in these cells.

Our immunohistological analysis of human first and third trimester placenta shows a comparable localization of $\alpha 8$ integrin, FN, and OPN in placental compartments at both time points. The localization of $\alpha 8$ integrin in the placental compartments argues for a possible role in trophoblast turnover and/or differentiation and in EVT invasion or anchorage.

We did not observe an association of $\alpha 8$ integrin expression levels and staining patterns with human placental pathologies, such as IUGR, PE, and HELLP, as was partly shown for its ligand OPN. ${ }^{64}$ Moreover, we did not observe a reduction in birth weight in our $\alpha 8$ integrin-deficient mice. This observation seems to argue against an important contribution of $\alpha 8$ integrin to placental function or, alternatively, might indicate effective compensatory mechanisms, which might substitute for a lack in $\alpha 8$ integrin expression. Human studies revealed that recessive mutations in the $\alpha 8$ integrin gene can lead to bilateral renal agenesis resulting in abortions ${ }^{65}$ which is in line with the findings in $\alpha 8$ integrin-deficient mice. ${ }^{23}$ Human placentas were not investigated for mutations in the $\alpha 8$ integrin gene thus far. One has to keep in mind, however, that the murine placenta is somewhat different in comparison with human placenta. ${ }^{50,66}$ Beyond their common hemochorial nature (trophoblast in contact with maternal blood), rodent placenta varies from human placenta due to its organization in junctional and labyrinthine zones (reviewed by Dilworth MR and Sibley ${ }^{50}$ ). Although the 


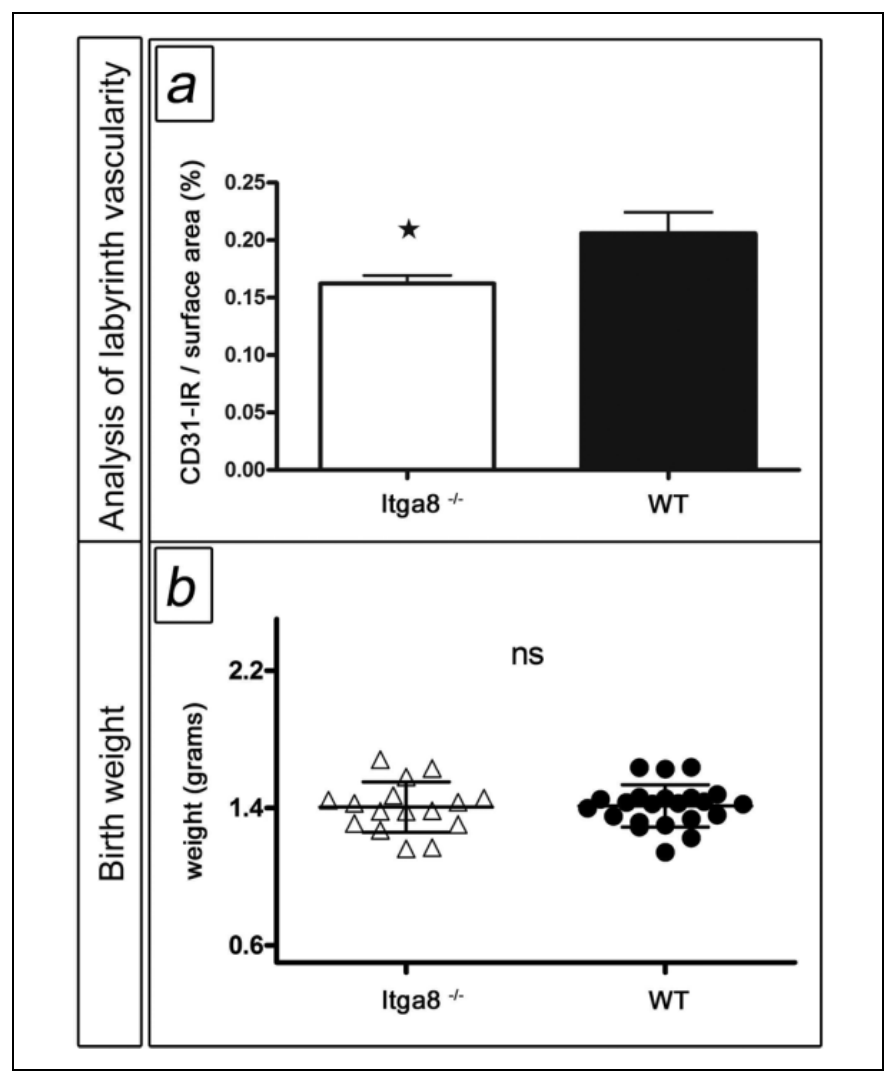

Figure 5. Labyrinth vascularity and neonatal body weights in $\alpha 8$ integrin-deficient mice. A, The quantitative analysis of the labyrinth vascularity via $C D 31$ staining (immunofluorescence with secondary antibody coupled to Cy 3 ) in $\alpha 8$ integrin-deficient (Itga ${ }^{-1-}$ ) and WT mice (representative of $n=5$, each). $\star P<.05$. B, The birth weights (mean $\pm \mathrm{SD}$ ) of $\alpha 8$ integrin-deficient (Itga $8^{-1-}$ ) and WT mice in grams ( $\mathrm{n}=22 \mathrm{WT}, \mathrm{n}=16 \mathrm{Itga}^{-1-}$, from 5 different litters). IR indicates immunoreactivity; SD, standard deviation; WT, wild-type.

Table 2. mRNA Expression Analyses of Mouse Placenta. ${ }^{a}$

\begin{tabular}{lcc}
\hline (Fold Change) & WT & Itga8 $^{-1-}$ \\
\hline Itga8 & $1.00 \pm 0.30$ & $0.0018 \pm 0.0011^{b}$ \\
Itga5 & $1.00 \pm 0.11$ & $0.87 \pm 0.12^{\mathrm{c}}$ \\
ItgaV & $1.00 \pm 0.13$ & $0.83 \pm 0.15^{\mathrm{c}}$ \\
Itga2 & $1.00 \pm 0.07$ & $1.02 \pm 0.18^{\mathrm{c}}$ \\
Itga6 & $1.00 \pm 0.14$ & $1.04 \pm 0.13^{\mathrm{c}}$ \\
Itgal & $1.00 \pm 0.08$ & $1.00 \pm 0.05^{\mathrm{c}}$ \\
\hline
\end{tabular}

Abbreviations: mRNA, messenger RNA; WT, wild-type.

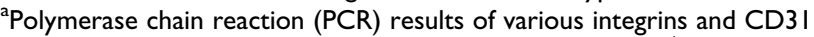
obtained from total placentas of $\alpha 8$ integrin-deficient (Itga $8^{-1-}, n=5$ ) and WT $(n=7)$ mice. Data are given as fold-change compared to WT (mean \pm standard error of the mean [SEM]).

${ }^{\mathrm{b}} \mathrm{P}<.001$.

'Not significant.

former is associated with endocrine function, the latter comprises the maternofetal exchange layer. The trophoblast cells of the junctional zone (ST, glycogen, and GCs) share similarities with human EVT, ${ }^{67-70}$ whereas the rodent labyrinth resembles the human SCT as maternofetal exchange surface.
Interestingly, the architecture of the rodent labyrinth is hemotri-chorial, compared to the hemo-mono-chorial SCT of the human placenta, consisting of sinusoidal trophoblast GCs and 2 syncytial trophoblast layers. ${ }^{50,71,72}$ Comparison of placentas among the studied species revealed that only mouse placenta shared syncytial $\alpha 8$ integrin expression with human placenta. Although in humans and rats the syncytium remained FN negative, the murine syncytium showed a positive FN immunoreactivity. This might suggest that in the human placenta, syncytial $\alpha 8$ integrin possibly interacts with binding partners other than FN, such as nephronectin, ${ }^{73}$ tenascin, ${ }^{74}$ or vitronectin. ${ }^{75}$

In analogy to the human EVT, the GCs of both rat and mouse showed a positive $\alpha 8$ integrin, FN, and OPN staining, arguing for an interaction of these proteins in the junctional zone. An important function of FN is its ability to strengthen EVT basal plate adhesion and its support of trophoblast migratory function ${ }^{76}$ in embryogenesis. ${ }^{77}$

To study a possible role for $\alpha 8$ integrin in the development of the murine placenta, we analyzed placentas from homozygous $\alpha 8$ integrin-deficient mice. We detected a reduced CD31 immunoreactivity, indicative of alterations of labyrinth layer vascularity. Welser et $\mathrm{al}^{16}$ showed that $\alpha 7$ integrin null embryos exhibit vascular smooth muscle cell defects that result in a reduced vascularity of the labyrinth layer and contribute to the partial embryonic lethality. Similarly, $\alpha 8$ integrin, which is abundantly expressed in smooth muscle of the vasculature, ${ }^{78}$ regulates smooth muscle function ${ }^{28,60}$ and underexpression of $\alpha 8$ integrin results in an increased susceptibility to develop atherosclerotic lesions and arterial remodeling. ${ }^{27}$ However, the observed change in placental vascularity seems to be only a subtle alteration, as the expression of the endothelial cell marker CD31 was not different in mice lacking the $\alpha 8$ integrin. Moreover, the placental deficiency for $\alpha 8$ integrin seems to be largely compensated for, as it was not reflected by a reduction in birth weight in these animals. There are some candidate integrins, which might compensate for the lack of $\alpha 8$ integrin. $\alpha 5$ integrin and $\alpha \mathrm{v}$ integrin are integrins that share many characteristics with $\alpha 8$ integrin and might thus maintain tissue integrity in placentas with a deficiency for $\alpha 8$ integrin. On the other hand, $\alpha 8$ integrindeficient renal mesangial cells upregulate the expression of $\alpha 2$ integrin and $\alpha 6$ integrin. $^{79,80}$ Thus, these integrins might also be candidates for compensatory effects. ${ }^{26}$ Expression analysis of these integrins, however, did not reveal differences in $\alpha 8$ integrin-deficient and WT placentas, arguing against a contribution of these integrins to any compensation for the lack of $\alpha 8$ integrin.

Taken together, we describe trophoblast $\alpha 8$ integrin expression in human and rodent placenta. We demonstrate species-specific expression patterns of $\alpha 8$ integrin and its binding partners FN and OPN in the syncytium, yet homogenous coexpression in the EVT and GC among the different species. As signaling via $\alpha 8$ integrin is known to be involved in several cell functions such as cell proliferation, apoptosis or adhesion, and migration, it is conceivable that $\alpha 8$ integrin 
contributes to normal placental development and helps to maintain structural integrity of the placenta. Given the fact that $\alpha 8$ integrin deficiency does not lead to an adverse fetal outcome, we suspect that placental $\alpha 8$ integrin function can be compensated for. However, lack of placental $\alpha 8$ integrin might nevertheless predispose to placental failure following a second hit (eg, infection, malnutrition), as shown for other organs such as the kidney and vasculature ${ }^{27,81}$ in $\alpha 8$ integrin-deficient mice.

\section{Acknowledgments}

The authors thank R. Wachtveitl and I. Winterfeld for expert technical assistance and the research staff of the Departments of Gynaecology and Obstetrics and Pathology at the University of Erlangen-Nürnberg for their kind collaboration. The authors further thank S. Fahlbusch for revising the manuscript. Establishment of staining procedures and respective data acquisition was performed by Sebastian Herdl in fulfillment of the requirements for obtaining the degree "Dr med." at the Department of Pediatrics and Adolescent Medicine, FriedrichAlexander-University of Erlangen-Nürnberg, Germany.

\section{Declaration of Conflicting Interests}

The author(s) declared no potential conflicts of interest with respect to the research, authorship, and/or publication of this article.

\section{Funding}

The author(s) received no financial support for the research, authorship, and/or publication of this article.

\section{Supplementary Material}

Supplementary material is available for this article online.

\section{References}

1. Huppertz B. Placental pathology in pregnancy complications. Thromb Res. 2011;127(suppl 3):S96-S99.

2. Huppertz B. Trophoblast differentiation, fetal growth restriction and preeclampsia. Pregnancy Hypertens. 2011;1(1):79-86.

3. Huppertz B. Placental origins of preeclampsia: challenging the current hypothesis. Hypertension. 2008;51(4):970-975.

4. Cetin I, Foidart JM, Miozzo M, et al. Fetal growth restriction: a workshop report. Placenta. 2004;25(8-9):753-757.

5. Abramovici D, Friedman SA, Mercer BM, Audibert F, Kao L, Sibai BM. Neonatal outcome in severe preeclampsia at 24 to 36 weeks' gestation: does the HELLP (hemolysis, elevated liver enzymes, and low platelet count) syndrome matter? Am J Obstet Gynecol. 1999;180(1 Pt 1):221-225.

6. Howe A, Aplin AE, Alahari SK, Juliano RL. Integrin signaling and cell growth control. Curr Opin Cell Biol. 1998;10(2): 220-231.

7. Ryu JS, Majeska RJ, Ma Y, LaChapelle L, Guller S. Steroid regulation of human placental integrins: suppression of alpha2 integrin expression in cytotrophoblasts by glucocorticoids. Endocrinology. 1999;140(9):3904-3908.

8. Humphries MJ. Integrin structure. Biochem Soc Trans. 2000; 28(4):311-339.

9. Schwartz MA. Integrin signaling revisited. Trends Cell Biol. 2001;11(12):466-470.
10. Hynes RO. Integrins: bidirectional, allosteric signaling machines. Cell. 2002;110(6):673-687.

11. Ruegg C, Mariotti A. Vascular integrins: pleiotropic adhesion and signaling molecules in vascular homeostasis and angiogenesis. Cell Mol Life Sci. 2003;60(6):1135-1157.

12. Giancotti FG, Ruoslahti E. Integrin signaling. Science. 1999; 285(5430):1028-1032.

13. Aplin JD, Jones CJ, Harris LK. Adhesion molecules in human trophoblast-a review. I. Villous trophoblast. Placenta. 2009; 30(4):293-298.

14. Harris LK, Jones CJ, Aplin JD. Adhesion molecules in human trophoblast - a review. II. extravillous trophoblast. Placenta. 2009;30(4):299-304.

15. Yang JT, Rayburn H, Hynes RO. Cell adhesion events mediated by alpha 4 integrins are essential in placental and cardiac development. Development. 1995;121(2):549-560.

16. Welser JV, Lange ND, Flintoff-Dye N, Burkin HR, Burkin DJ. Placental defects in $\alpha 7$ integrin null mice. Placenta. 2007;28(1112):1219-1228.

17. Bossy B, Bossy-Wetzel E, Reichardt LF. Characterization of the integrin alpha 8 subunit: a new integrin beta 1-associated subunit, which is prominently expressed on axons and on cells in contact with basal laminae in chick embryos. EMBO J. 1991;10(9):2375-2385.

18. Schnapp LM, Hatch N, Ramos DM, Klimanskaya IV, Sheppard $\mathrm{D}$, Pytela R. The human integrin alpha 8 beta 1 functions as a receptor for tenascin, fibronectin, and vitronectin. $J$ Biol Chem. 1995;270(39):23196-23202.

19. Brandenberger R, Schmidt A, Linton J, et al. Identification and characterization of a novel extracellular matrix protein nephronectin that is associated with integrin alpha8 beta1 in the embryonic kidney. J Cell Biol. 2001;154(2):447-458.

20. Denda S, Reichardt LF, Muller U. Identification of osteopontin as a novel ligand for the integrin alpha8 beta1 and potential roles for this integrin-ligand interaction in kidney morphogenesis. Mol Biol Cell. 1998;9(6):1425-1435.

21. Chen CP, Aplin JD. Placental extracellular matrix: gene expression, deposition by placental fibroblasts and the effect of oxygen. Placenta. 2003;24(4):316-325.

22. Johnson GA, Burghardt RC, Bazer FW, Spencer TE. Osteopontin: roles in implantation and placentation. Biol Reprod. 2003;69(5): 1458-1471.

23. Muller U, Wang D, Denda S, Meneses JJ, Pedersen RA, Reichardt LF. Integrin alpha8beta1 is critically important for epithelialmesenchymal interactions during kidney morphogenesis. Cell. 1997;88(5):603-613.

24. Littlewood Evans A, Muller U. Stereocilia defects in the sensory hair cells of the inner ear in mice deficient in integrin alpha8beta1. Nat Genet. 2000;24(4):424-428.

25. Hartner A, Cordasic N, Klanke B, Muller U, Sterzel RB, Hilgers KF. The alpha8 integrin chain affords mechanical stability to the glomerular capillary tuft in hypertensive glomerular disease. Am J Pathol. 2002;160(3):861-867.

26. Hartner A, Marek I, Cordasic N, et al. Glomerular regeneration is delayed in nephritic alpha 8-integrin-deficient mice: contribution of alpha 8-integrin to the regulation of mesangial cell apoptosis. Am J Nephrol. 2008;28(1):168-178. 
27. Menendez-Castro C, Cordasic N, Neureiter D, et al. Underexpression of alpha8 integrin aggravates experimental atherosclerosis. J Pathol. 2015;236(1):5-16.

28. Zargham R, Thibault G. Alpha 8 integrin expression is required for maintenance of the smooth muscle cell differentiated phenotype. Cardiovasc Res. 2006;71(1):170-178.

29. Zargham R, Touyz RM, Thibault G. alpha 8 Integrin overexpression in de-differentiated vascular smooth muscle cells attenuates migratory activity and restores the characteristics of the differentiated phenotype. Atherosclerosis. 2007;195(2):303-312.

30. Zargham R, Wamhoff BR, Thibault G. RNA interference targeting alpha8 integrin attenuates smooth muscle cell growth. FEBS Lett. 2007;581(5):939-943.

31. Benoit YD, Lussier C, Ducharme PA, et al. Integrin alpha8beta1 regulates adhesion, migration and proliferation of human intestinal crypt cells via a predominant RhoA/ROCK-dependent mechanism. Biol Cell. 2009;101(12):695-708.

32. Bieritz B, Spessotto P, Colombatti A, Jahn A, Prols F, Hartner A. Role of alpha8 integrin in mesangial cell adhesion, migration, and proliferation. Kidney Int. 2003;64(1):119-127.

33. Benoit YD, Larrivee JF, Groulx JF, Stankova J, Vachon PH, Beaulieu JF. Integrin alpha8beta1 confers anoikis susceptibility to human intestinal epithelial crypt cells. Biochem Biophys Res Commun. 2010;399(3):434-439.

34. Farias E, Lu M, Li X, Schnapp LM. Integrin alpha8beta1fibronectin interactions promote cell survival via PI3 kinase pathway. Biochem Biophys Res Commun. 2005;329(1): 305-311.

35. World Medical Association. World Medical Association Declaration of Helsinki: ethical principles for medical research involving human subjects. JAMA. 2013;310(20):2191-2194.

36. Fahlbusch FB, Dawood Y, Hartner A, et al. Cullin 7 and Fbxw 8 expression in trophoblastic cells is regulated via oxygen tension: implications for intrauterine growth restriction? J Matern Fetal Neonatal Med. 2012;25(11):2209-2215.

37. Fahlbusch FB, Ruebner M, Huebner H, et al. Trophoblast expression dynamics of the tumor suppressor gene gastrokine 2. Histochem Cell Biol. 2015;144(3):281-291.

38. Langbein M, Strick R, Strissel PL, et al. Impaired cytotrophoblast cell-cell fusion is associated with reduced Syncytin and increased apoptosis in patients with placental dysfunction. Mol Reprod Dev. 2008;75(1):175-183.

39. Fahlbusch FB, Ruebner M, Huebner H, et al. The tumor suppressor gastrokine-1 is expressed in placenta and contributes to the regulation of trophoblast migration. Placenta. 2013;34(11): 1027-1035.

40. Ruebner M, Strissel PL, Langbein M, et al. Impaired cell fusion and differentiation in placentas from patients with intrauterine growth restriction correlate with reduced levels of HERV envelope genes. J Mol Med (Berl). 2010;88(11):1143-1156.

41. Kliman HJ, Nestler JE, Sermasi E, Sanger JM, Strauss JF III, Purification, characterization, and in vitro differentiation of cytotrophoblasts from human term placentas. Endocrinology. 1986; 118(4):1567-1582.

42. Kontopoulos EV, Chandras C, Karalis K. Terbutaline inhibits corticotropin-releasing hormone $(\mathrm{CRH})$ expression in human trophoblast cells. J Matern Fetal Neonatal Med. 2006;19(11): 735-739.

43. Frank GR, Brar AK, Cedars MI, Handwerger S. Prostaglandin E2 enhances human endometrial stromal cell differentiation. Endocrinology. 1994;134(1):258-263.

44. Lockwood CJ, Yen CF, Basar M, et al. Preeclampsia-related inflammatory cytokines regulate interleukin-6 expression in human decidual cells. Am J Pathol. 2008;172(6):1571-1579.

45. Untergasser A, Cutcutache I, Koressaar T, et al. Primer3-new capabilities and interfaces. Nucleic Acids Res. 2012;40(15):e115.

46. Hartner A, Hilgers KF, Bitzer M, Veelken R, Schocklmann HO. Dynamic expression patterns of transforming growth factorbeta(2) and transforming growth factor-beta receptors in experimental glomerulonephritis. J Mol Med (Berl). 2003;81(1):32-42.

47. Hartner A, Porst M, Gauer S, Prols F, Veelken R, Hilgers KF. Glomerular osteopontin expression and macrophage infiltration in glomerulosclerosis of DOCA-salt rats. Am J Kidney Dis. 2001; 38(1):153-164.

48. Hartner A, Schocklmann H, Prols F, Muller U, Sterzel RB. Alpha8 integrin in glomerular mesangial cells and in experimental glomerulonephritis. Kidney Int. 1999;56(4):1468-1480.

49. Frese S, Ruebner M, Suhr F, et al. Long-term endurance exercise in humans stimulates cell fusion of myoblasts along with fusogenic endogenous retroviral genes in vivo. PLoS One. 2015;10(7): e0132099.

50. Dilworth MR, Sibley CP. Review: transport across the placenta of mice and women. Placenta. 2013;34(suppl):S34-S39.

51. Rossant J, Cross JC. Placental development: lessons from mouse mutants. Nat Rev Genet. 2001;2(7):538-548.

52. Watson ED, Cross JC. Development of structures and transport functions in the mouse placenta. Physiology (Bethesda). 2005;20: 180-193.

53. Schneider CA, Rasband WS, Eliceiri KW. NIH Image to ImageJ: 25 years of image analysis. Nat Methods. 2012;9(7):671-675.

54. Kang JA, Zhou Y, Weis TL, et al. Osteopontin regulates actin cytoskeleton and contributes to cell proliferation in primary erythroblasts. J Biol Chem. 2008;283(11):6997-7006.

55. Damsky CH, Fitzgerald ML, Fisher SJ. Distribution patterns of extracellular matrix components and adhesion receptors are intricately modulated during first trimester cytotrophoblast differentiation along the invasive pathway, in vivo. J Clin Invest. 1992; 89(1):210-222.

56. Fisher SJ, Cui TY, Zhang L, et al. Adhesive and degradative properties of human placental cytotrophoblast cells in vitro. $J$ Cell Biol. 1989;109(2):891-902.

57. Zhou Y, Damsky CH, Chiu K, Roberts JM, Fisher SJ. Preeclampsia is associated with abnormal expression of adhesion molecules by invasive cytotrophoblasts. J Clin Invest. 1993; 91(3):950-960.

58. Zhou Y, Damsky CH, Fisher SJ. Preeclampsia is associated with failure of human cytotrophoblasts to mimic a vascular adhesion phenotype. One cause of defective endovascular invasion in this syndrome? J Clin Invest. 1997;99(9):2152-2164.

59. Zhou Y, Fisher SJ, Janatpour M, et al. Human cytotrophoblasts adopt a vascular phenotype as they differentiate. A strategy for successful endovascular invasion? J Clin Invest. 1997;99(9):2139-2151. 
60. Zargham R, Thibault G. alpha8beta1 Integrin expression in the rat carotid artery: involvement in smooth muscle cell migration and neointima formation. Cardiovasc Res. 2005;65(4):813-822.

61. Kabir-Salmani M, Shiokawa S, Akimoto Y, Sakai K, Iwashita M. The role of alpha(5)beta(1)-integrin in the IGF-I-induced migration of extravillous trophoblast cells during the process of implantation. Mol Hum Reprod. 2004;10(2):91-97.

62. Feinberg RF, Kliman HJ, Lockwood CJ. Is oncofetal fibronectin a trophoblast glue for human implantation? Am J Pathol. 1991; 138(3):537-543.

63. Bischof P, Haenggeli L, Campana A. Gelatinase and oncofetal fibronectin secretion is dependent on integrin expression on human cytotrophoblasts. Hum Reprod. 1995;10(3):734-742.

64. Gabinskaya T, Salafia CM, Gulle VE, Holzman IR, Weintraub AS. Gestational age-dependent extravillous cytotrophoblast osteopontin immunolocalization differentiates between normal and preeclamptic pregnancies. Am J Reprod Immunol. 1998; 40(5):339-346.

65. Humbert C, Silbermann F, Morar B, et al. Integrin alpha 8 recessive mutations are responsible for bilateral renal agenesis in humans. Am J Hum Genet. 2014;94(2):288-294.

66. Davies J, Glasser SR. Histological and fine structural observations on the placenta of the rat. Acta Anat (Basel). 1968;69(4):542-608.

67. Hemberger M, Cross JC. Genes governing placental development. Trends Endocrinol Metab. 2001;12(4):162-168.

68. Cross JC, Baczyk D, Dobric N, et al. Genes, development and evolution of the placenta. Placenta. 2003;24(2-3):123-130.

69. Georgiades P, Ferguson-Smith AC, Burton GJ. Comparative developmental anatomy of the murine and human definitive placentas. Placenta. 2002;23(1):3-19.

70. Simmons DG, Cross JC. Determinants of trophoblast lineage and cell subtype specification in the mouse placenta. Dev Biol. 2005; 284(1):12-24.
71. Henke C, Ruebner M, Faschingbauer F, et al. Regulation of murine placentogenesis by the retroviral genes Syncytin-A, SyncytinB and Peg10. Differentiation. 2013;85(4-5):150-160.

72. Simmons DG, Natale DR, Begay V, Hughes M, Leutz A, Cross JC. Early patterning of the chorion leads to the trilaminar trophoblast cell structure in the placental labyrinth. Development. 2008; 135(12):2083-2091.

73. Huang JT, Lee V. Identification and characterization of a novel human nephronectin gene in silico. Int J Mol Med. 2005;15(4):719-724.

74. Castellucci M, Classen-Linke I, Muhlhauser J, Kaufmann P, Zardi L, Chiquet-Ehrismann R. The human placenta: a model for tenascin expression. Histochemistry. 1991;95(5):449-458.

75. Shen J, Ma X, Yi F, Rasul A, Cui M, Ma T. Increased expression levels of vitronectin in the maternal-fetal interface of placenta in early-onset severe preeclampsia. Mol Med Rep. 2013;7(1):53-58.

76. Earl U, Estlin C, Bulmer JN. Fibronectin and laminin in the early human placenta. Placenta. 1990;11(3):223-231.

77. Kornblihtt AR, Pesce CG, Alonso CR, et al. The fibronectin gene as a model for splicing and transcription studies. FASEB J. 1996; 10(2):248-257.

78. Schnapp LM, Breuss JM, Ramos DM, Sheppard D, Pytela R. Sequence and tissue distribution of the human integrin alpha 8 subunit: a beta 1-associated alpha subunit expressed in smooth muscle cells. J Cell Sci. 1995;108(2):537-544.

79. Marek I, Volkert G, Jahn A, et al. Lack of alpha8 integrin leads to morphological changes in renal mesangial cells, but not in vascular smooth muscle cells. BMC Cell Biol. 2010;11:102.

80. Haas CS, Amann K, Schittny J, Blaser B, Muller U, Hartner A. Glomerular and renal vascular structural changes in alpha8 integrin-deficient mice. J Am Soc Nephrol. 2003;14(9):2288-2296.

81. Hartner A, Dotsch J. Lessons in congenital and acquired renal disease from alpha 8 integrin mutant mice. Pediatr Nephrol. 2002;17(11):882-888. 